\title{
Fitting the Fully Coupled ORM for the Fermilab Booster *
}

\author{
X. Huang ${ }^{\dagger}$, S. Y. Lee, Indiana University, Bloomington, IN 47405, USA \\ Eric Prebys , Chuck Ankenbrandt, Fermilab, Batavia, IL 60510, USA

\section{THE FITTING MODEL AND ALGORITHM}

\section{Abstract}

The orbit response matrix (ORM) method [1] is applied to model the Fermilab Booster with parameters such as the BPM gains and rolls, and parameters in the lattice model, including the gradient errors and magnets rolls. We found that the gradients and rolls of the adjacent combinedfunction magnets were deeply correlated, preventing full determination of the model parameters. Suitable constraints of the parameters were introduced to guarantee an unique, equivalent solution. Simulations show that such solution preserves proper combinations of the adjacent parameters. The result shows that the gradient errors of combined-function magnets are within design limits.

\section{INTRODUCTION}

The Fermilab Booster is a fast-cycling synchrotron which accelerates proton beams from $400 \mathrm{MeV}$ to $8 \mathrm{GeV}$. Its performance is critical for many Fermilab experiments. Among the many efforts to improve the Booster, we were trying to build a realistic lattice model with beam-based measurements, such as the orbit response matrix (ORM) method.

The ORM method, successfully applied to many electron storage rings, is a powerful tool for accelerator lattice modeling. However its application to fast-ramping, proton synchrotrons such as the Fermilab Booster is more difficult because of the reduced orbit stability and precision of orbit measurements. In our study we found another difficulty is the correlation between the model parameters of the adjacent magnets. Suitable constraints have to used to obtain an unique solution.

The Booster has 24 identical periods. Each period has four combined function magnets and two straight sections with a layout of $\{$ O FU DU OOO DD FD O $\}$, where OOO stands for the long straight section, $\mathrm{O}$ for a half of the short straight section, FU for upstream focusing magnet and DU for upstream defocussing magnet, etc. In each straight section there are one BPM, which measures both horizontal and vertical orbits, one horizontal trim (a steering dipole magnet, or a kicker) and one vertical trim. Note that there is only one BPM in every two magnet elements. The full ORM is $96 \times 96$ in dimension.

\footnotetext{
* Work supported by grants from DE-AC02-76CH03000, DOE DEFG02-92ER40747 and NSF PHY-0244793

†xiahuang@fnal.gov
}

The model ORM is computed with the transfer matrix of the lattice model [2]. Suppose $\mathbf{T}$ is the one-turn transfer matrix at a trim and $\mathbf{I}$ is a $4 \times 4$ identify matrix, the orbit deviation $x_{0}$ and $z_{0}$ at the trim's location due to an horizontal kick $\theta_{x}$ of the trim can be found by $\left(x_{0}, x_{0}^{\prime}, z_{0}, z_{0}^{\prime}\right)^{T}=$ $(\mathbf{T}-\mathbf{I})^{-1}\left(0,-\theta_{x}, 0,0\right)^{T}$. The ORM elements can then be calculated using the transfer matrices between the trim and BPMs. The same can be done with vertical trims.

The measured ORM needs to be corrected to take into account the imperfections of the measurement systems, e.g. BPMs and trims. The correction parameters include the gains and rolls of BPMs, the gains and rolls of both horizontal and vertical trims, the momentum deviation due to horizontal trims. The additional horizontal orbit changes caused by the momentum deviation are subtracted from the measured orbit to get the correct ORM. The parameters in the lattice model are the gradient errors and rolls of all 96 combined-function magnets. There are a total of 576 fitting parameters.

The difference between the model and measured ORM is characterized by the residual vector $\mathbf{r}$ which is a column vector containing all elements of $\left(M_{i j}^{\text {act }}-M_{i j}^{\text {model }}\right) / \sigma_{i j}$ and the objective function $\chi^{2}=\mathbf{r}^{T} \mathbf{r}$. The betatron tunes are included by extending the residual vector. The dispersion functions can also be included. The fitting parameters are put in a column vector $\alpha$. The Levenberg-Marquardt method [4] is used to solve the nonlinear least-square problem of $f(\alpha)=\chi^{2}$. For each iteration the Jacobian matrix $\mathbf{J}=\frac{\partial \mathbf{r}}{\partial \alpha}$ is computed and the advance of $\alpha$ is found by solving the following equation

$$
\left(\mathbf{J}^{T} \mathbf{J}+\lambda \mathbf{I}\right) \Delta \alpha=-\mathbf{J}^{T} \mathbf{r}
$$

instead of solving $\mathbf{J} \Delta \alpha=-\mathbf{r}$ as suggested in Ref. [1], where $\mathbf{I}$ is the identity matrix and $\lambda$ is an adjustable parameter. This approach is more robust. It is also faster because the matrix on the left side of Eq. 1 has a smaller size than $\mathbf{J}$. The error bars of the fitting parameters are estimated by computing the covariance matrix $\mathbf{C}=\mathbf{J}^{T} \mathbf{J}$ and then $\sigma_{i}=\sqrt{C_{i i}}$ for the $i$ 'th parameter.

The above fitting scheme is correct in principle but would not work even for simulated noise-free ORM because the Fermilab Booster beam detection system (1 BPM in every 2 magnet elements) is not sufficient for an unique solution. In one simulation, the "measured" ORM is generated by setting the gradient error of one magnet to $\Delta K_{1}=$ $0.002 \mathrm{~m}^{-2}$, or $4 \%$ of the nominal quadrupole gradient. The algorithm reduces $\chi^{2}$ down to zero efficiently but does not converge to the expected solution. The gradients of the 


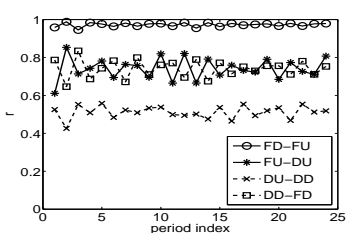

(a) quads

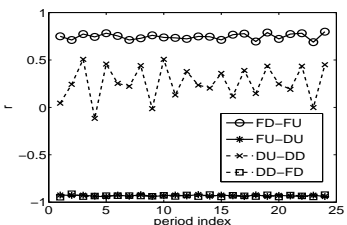

(b) rolls

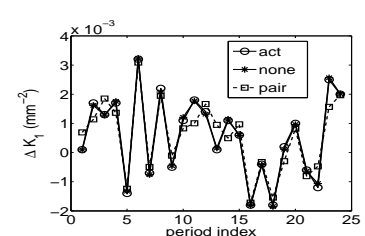

(a) quads

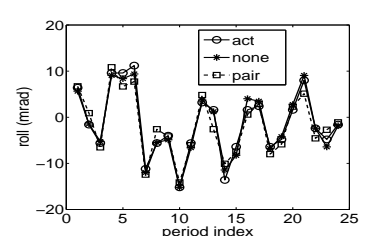

(b) rolls
Figure 1: The correlation coefficients for model parameters of neighboring magnets. (a) The gradient errors. (b) The magnet rolls.

immediate neighboring magnets also pop up and make up part of the contribution to $\chi^{2}$. The magnet rolls show similar behavior.

Such observations suggest correlations between model parameters of the adjacent magnets, i.e., changes of these parameters perturb the ORM in similar patterns. The correlation are illustrated in Fig. 1, which shows the correlation coefficients between the columns of the Jacobian matrix.

The gradients of neighboring focusing magnets (FD FU), the rolls of neighboring upstream magnets (FU-DU) or downstream magnets (DD-FD) have the deepest correlation. The correlation indicates that the ORM fitting problem is deficient and the model parameters cannot be determined individually. The solution is not well-constrained in some directions and tends to have big error bars.

Although the correlation prevents the full determination of the real Booster lattice from the ORM data, it is still desirable to have a definite solution of the fitting problem, which can be obtained by imposing proper constraints. For example, we may require a solution with minimum Euclidean norm. A more efficient way is to limit the drifting along the un-constrained directions by minimizing certain combinations of the correlated parameters. These combinations include: (1) $(\theta(\mathrm{FU}, i)+\theta(\mathrm{DU}, i)) / \sigma_{\theta}$, rolls of upstream magnets; (2) $(\theta(\mathrm{DD}, i)+\theta(\mathrm{FD}, i)) / \sigma_{\theta}$, rolls of downstream magnets; (3) $(\theta(\mathrm{FD}, i)-\theta(\mathrm{FU}, i+1)) / \sigma_{\theta}$, rolls of neighboring focusing magnets; (4) $\left(\Delta K_{1}(\mathrm{FD}, i)-\right.$ $\left.\Delta K_{1}(\mathrm{FU}, i+1)\right) / \sigma_{K_{1}}$, quads of neighboring focusing magnets; (5) $\left(\Delta K_{1}(\mathrm{FU}, i)-\Delta K_{1}(\mathrm{DU}, i)\right) / \sigma_{K_{1}}$, quads of upstream magnets; (6) $\left(\Delta K_{1}(\mathrm{DD}, i)-\Delta K_{1}(\mathrm{FD}, i)\right) / \sigma_{K_{1}}$, quads of downstream magnets, where $i$ denotes the period number, $\sigma_{K_{1}}=0.00055 \mathrm{~m}^{-2}$ and $\sigma_{\theta}=5 \mathrm{mrad}$ are characteristic values to scale the parameters.

A comprehensive simulation was used to check the fitting schemes. The parameters were set to random values within a reasonable scope to generate the ORM. The constrained fitting converges to an unique solution and reduces $\chi^{2}$ from 32 to 0.01 . The solution is much less sensitive to random noises. Assuming error sigma of matrix elements of $1.0 \mathrm{~m} / \mathrm{rad}$, the average error sigmas of gradients are $0.0042 \mathrm{~m}^{-2}$ without constraints and $2.5 \times 10^{-4} \mathrm{~m}^{-2}$ with constraints, while for rolls they are $78.7 \mathrm{mrad}$ and $1.7 \mathrm{mrad}$,
Figure 2: Comparisons of the fitting results with ("pair") or without ("none") constraints to the expected solution ("act"). (a) $\Delta K_{1}(\mathrm{FD})+\Delta K_{1}(\mathrm{FU})$, (b) $\theta(\mathrm{FU})-\theta(\mathrm{DU})$.

respectively. The constrained directions are faithfully recovered by both methods, as shown in Fig. 2.

\section{APPLICATION TO EXPERIMENTAL DATA}

Each ORM element is measured by applying different kick angles and measuring the beam orbits. The slope of orbit - kick angle is obtained by linear fitting and is turned to ORM element according to magnet specifications. The uncertainty levels of the elements are estimated using the residual $\chi^{2}$ of the linear fittings. The maximum kick angles are $0.58 \mathrm{mrad}$ for horizontal trims and $0.34 \mathrm{mrad}$ for vertical trims, resulting in maximum orbit changes of $8.0 \mathrm{~mm}$ and $3.0 \mathrm{~mm}$, respectively. The average error sigma's are $0.33 \mathrm{~m} / \mathrm{rad}$ for $\mathbf{M}_{x x}, 0.54 \mathrm{~m} / \mathrm{rad}$ for $\mathbf{M}_{x z}, 0.07 \mathrm{~m} / \mathrm{rad}$ for $\mathbf{M}_{z x}$ and $0.15 \mathrm{~m} / \mathrm{rad}$ for $\mathbf{M}_{z z}$. The vertical blocks have better precision because the vertical orbit has less cycleto-cycle variations than the horizontal orbit. The betatron tunes are measured by BPM turn-by-turn data. The dispersion functions are also measured. They are both included in the fitting by extending the residual vector. The dispersion terms are $\left(D_{i}^{\text {meas }}-D_{i}^{\text {model }}\right) /\left(b_{h, i} \sigma_{D, i}\right)$, where $b_{h, i}$ is horizontal BPM gain and $\sigma_{D, i}$ is error sigma for dispersion measurements. The inclusion of dispersion function would de-couple the BPM gains and kicker gains [1].

The Booster model has all up-to-date information, including the experimental settings of the trim quadrupoles and skew quadrupoles. The ORM data are taken at $0.9 \mathrm{~ms}$ after injection, corresponding to kinetic energy $0.41 \mathrm{GeV}$. The constrained fitting is applied, which reduces the normalized $\chi^{2}$ from 76.0 to 2.5 .

The $\chi^{2}$ contribution of each type of parameters is evaluated by setting all parameters to their fitted values except for that type, which are set to the default. We found that the major contributors are magnet rolls, kick-induced momentum deviation, vertical BPM gains and the gradient errors. The model ORMs and dispersion functions gain good agreements with their measured counterparts through the fitting process. Figures 3 and 4 compare the model result with the measured data before and after the ORM modeling for the dispersion function and the ORM row for one BPM. 
